
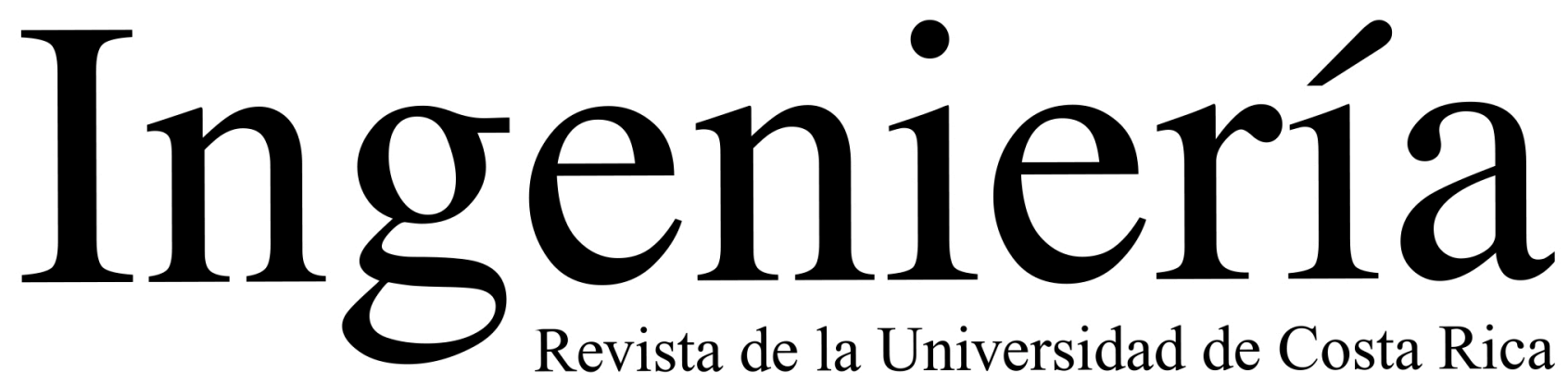

Revista de la Universidad de Costa Rica JULIO/DICIEMBRE 2021 - VOLUMEN 31 (2)

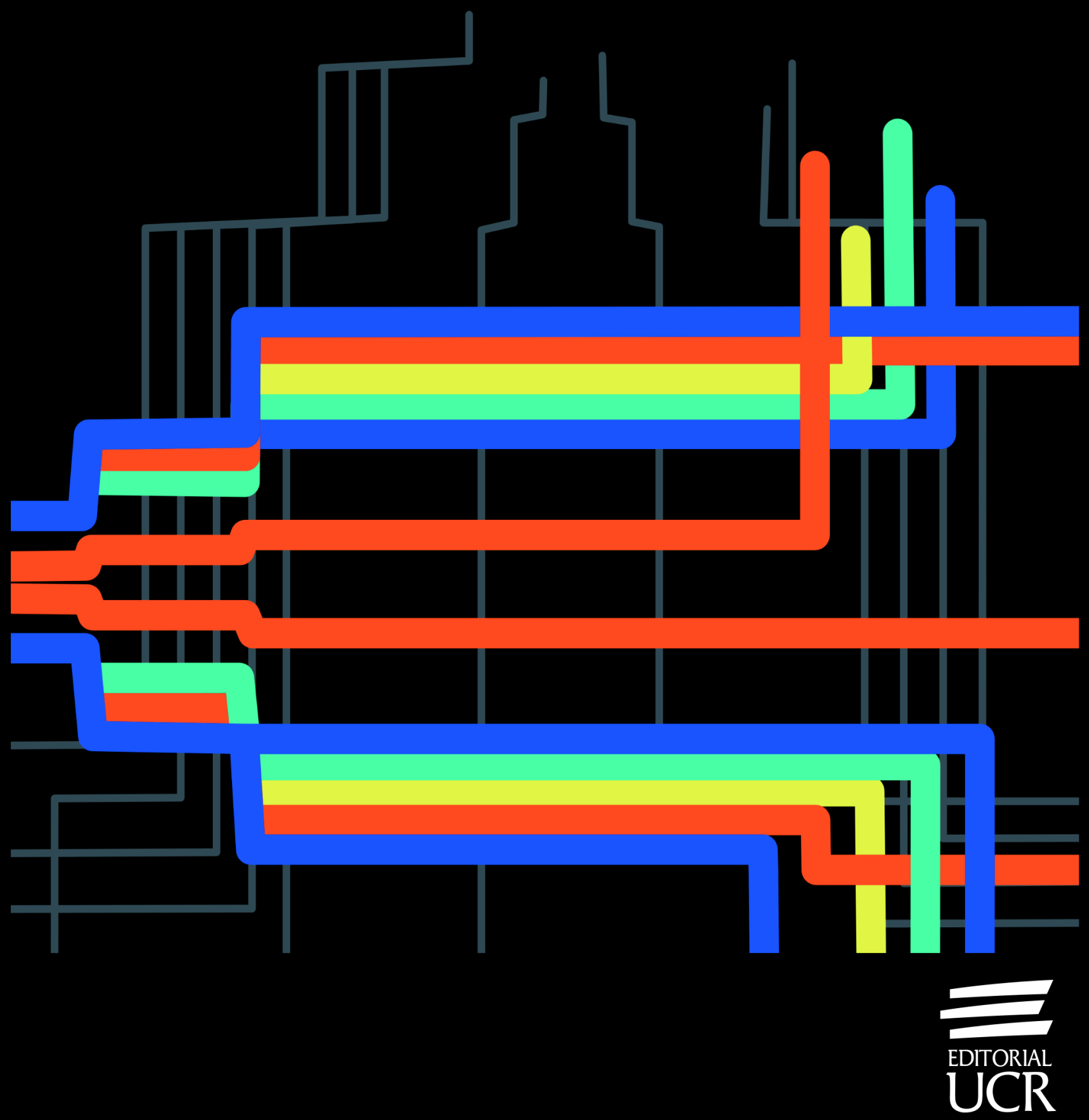




\title{
Incertidumbre asociada con la indicación de los instrumentos para pesar verificados
}

\section{Uncertainty associated with the indication of verified weighing instruments}

\author{
Luis Omar Becerra \\ Centro Nacional de Metrología, Ciudad de Querétaro, México \\ Email: lbecerra@cenam.mx, \\ ORCID:https://orcid.org/0000-0002-0530-5618 \\ Luis Manuel Peña \\ Centro Nacional de Metrología, Ciudad de Querétaro, México \\ Email:lpena@cenam.mx \\ ORCID:https://orcid.org/0000-0002-6960-8140
}

Recibido: 14 de diciembre $2020 \quad$ Aceptado: 22 de febrero 2021

\section{Resumen}

En el presente trabajo se estima la incertidumbre asociada con la indicación en uso de los instrumentos para pesar el funcionamiento no automático, el cual ha sido verificado acorde con la recomendación internacional OIML R76. Esta recomendación de la Organización Internacional de Metrología Legal (OIML) sirve de base para la verificación de los instrumentos para pesar de funcionamiento no automático para la gran mayoría de los países; sin embargo, no se conoce de manera explícita el valor de incertidumbre relacionada con los resultados de los instrumentos verificados durante su uso.

Palabras clave:

Calibración, Incertidumbre, Instrumentos para pesar, Verificación

\begin{abstract}
In the present work the uncertainty associated with the indication in the use of verified non-automatic weighing instruments according to the international recommendation OIML R76, is estimated. This recommendation of the International Organization of Legal Metrology (OIML) serves as the basis for the verification for most of the countries, however, the uncertainty associated with results of verified instruments during its use are not known explicitly.
\end{abstract}

Keywords:

Calibration, Uncertainty, Verification, Weighing instruments 


\section{INTRODUCCIÓN}

Muchos productos son comercializados por su masa; asimismo, existen una gran cantidad de procesos que son controlados mediante mediciones de esta magnitud. Estas mediciones incluyen desde valores muy pequeños, de unos cuantos miligramos, hasta decenas o centenas de toneladas, cantidades medidas en instrumentos para pesar verificados de acuerdo con las leyes y normas particulares de cada país.

Tanto calibrar como verificar instrumentos forman parte de la confirmación metrológica en el contexto de la ISO 10012 [1], proceso que tiene en cuenta, por un lado, los requisitos metrológicos del cliente, y por otro lado las características metrológicas del instrumento de medición. Así, el resultado corresponde al estado de confirmación del equipo de medición. Usualmente, la verificación consiste en la confirmación del cumplimiento de determinados requisitos metrológicos por parte del instrumento de medición, características metrológicas del equipo de medición determinadas por calibración.

Respecto a los instrumentos para pesar de funcionamiento no automático, la verificación de estos se ha realizado durante mucho tiempo como una actividad muy relacionada con la economía de los países; esto con base en recomendaciones de la Organización Internacional de Metrología Legal OIML, en particular la OIML R76, donde una de sus primeras versiones surgió en 1992, un año antes de que se publicara la Guía para la expresión de la incertidumbre en mediciones, (GUM por sus siglas en inglés) en 1993 (versión vigente [9]). Por tanto, no se incluyó la incertidumbre en las pruebas recomendadas para la verificación en los instrumentos para pesar. El impacto y amplia aplicación de la OIML R76 ha sido tal que, en la versión actual (2006), las pruebas e incluso el error máximo permitido de los instrumentos no consideran la incertidumbre de medición asociada, al menos no de manera explícita.

Por otro lado, en 2007 la EA (European Co-operation for Accreditation) desarrolló un documento para armonizar la calibración de los instrumentos para pesar de funcionamiento no automático; este incluyó la evaluación de la incertidumbre asociada con la calibración de los instrumentos (incertidumbre asociada con el error de indicación en calibración), así como una guía para la estimación de la incertidumbre de las indicaciones de los instrumentos en uso. Posteriormente, este documento pasó al resguardo de EUROMET (European Collaboration in Measurement Standards) y cuya versión actual [7] ha sido adoptada por el Sistema Interamericano de Metrología SIM [6], actualmente en revisión para su actualización.

En México la OIML R 76:2006 [2] es la referencia de la Norma Oficial Mexicana NOM-010 $[3,4]$ utilizada para realizar la verificación de los Instrumentos para Pesar de Funcionamiento No Automático (IPFNA) que requieren control metrológico legal (Art. 124 Ley Infraestructura de la Calidad, [5]). La NOM-010 actualmente está en revisión para actualizarla a la versión 2006 de la OIML R76.

En la OIML R76 se establecen los errores máximos permitidos (emp) que los IPFNA deben cumplir de acuerdo a su clase de exactitud, que a su vez corresponden a características de su fabricación, como la capacidad máxima de pesada (Max) y la división de la escala de verificación $(e)$. 
Un usuario bien informado podría conocer cuál es el emp asociado con una indicación particular de un instrumento verificado. Sin embargo, la incertidumbre asociada con dicha indicación (o el intervalo de probabilidad en donde razonablemente podría encontrase el valor verdadero de la medida) no está disponible al usuario de manera explícita.

Por tanto, en este trabajo se presenta un análisis de la incertidumbre de medición asociada con la indicación de un instrumento para pesar verificado de acuerdo a la OIML R76, aplicando las guías y recomendaciones de la Guía para la calibración de los instrumentos para pesar de funcionamiento no automático [6,7].

Es importante señalar que tanto la calibración como la verificación de los instrumentos para pesar se realizan en valores de masa convencional [8]. En algunas ocasiones, el usuario puede solicitar que la calibración de su instrumento se realice en valores de masa.

\section{VERIFICACIÓN DE LOS IPFNA}

La verificación de los IPFNA de acuerdo con la OIML R76, consiste básicamente en:

- $\quad$ la clasificación y la determinación de los emp del IPFNA,

- la aplicación cargas de prueba al instrumento para pesar bajo condiciones especificadas,

- la determinación del error de indicación respecto a la masa convencional del patrón para el alcance del instrumento y para condiciones de repetibilidad y de carga excéntrica,

- la contrastación de los errores de indicación el instrumento contra los correspondientes emp del instrumento.

Las principales pruebas metrológicas que se le realizan a un instrumento para pesar en la verificación (subsecuente) son las correspondientes al error de indicación, al error de excentricidad, al error de repetibilidad, la de exactitud del cero entre otras. El instrumento no debe presentar errores de indicación mayores al emp correspondiente para cada prueba. A su vez, para la verificación se requieren pesas patrón calibradas, cuya clase de exactitud sea acorde con la clase de exactitud del instrumento a verificar [8]. De acuerdo a la OIML R76, los IPFNA se clasifican en cuatro clases: de acuerdo con su exactitud, de acuerdo con la división de la escala de verificación, número de divisiones de verificación y capacidad mínima del instrumento, ver Tabla 1.

Por otro lado, en la Tabla 2 se presentan los emp asociados con la indicación del instrumento en función de la carga expresada en divisiones de verificación. 
TABLA 1

CLASES DE EXACTITUD DE LOS IPFNA [2]

\begin{tabular}{|c|c|c|c|c|}
\hline \multirow[t]{2}{*}{$\begin{array}{l}\text { Clase de } \\
\text { exactitud }\end{array}$} & \multirow{2}{*}{$\begin{array}{c}\text { División de la escala de } \\
\text { verificación } \\
e\end{array}$} & \multicolumn{2}{|c|}{$\begin{array}{c}\text { Número de divisiones de } \\
\text { verificación } \\
n=\operatorname{Max} / e\end{array}$} & \multirow[t]{2}{*}{$\begin{array}{l}\text { Capacidad mínima } \\
\text { Min }\end{array}$} \\
\hline & & Mínimo & Máximo & \\
\hline Especial (I) & $0.001 \mathrm{~g} \leq e$ & 50000 & --- & $100 e$ \\
\hline Fina (II) & $\begin{array}{c}0.001 \mathrm{~g} \leq e \leq 0.05 \mathrm{~g} \\
0.1 \mathrm{~g} \leq e\end{array}$ & $\begin{array}{c}100 \\
5000\end{array}$ & $\begin{array}{l}100000 \\
100000\end{array}$ & $\begin{array}{l}20 e \\
50 e\end{array}$ \\
\hline Media (III) & $\begin{array}{c}0.1 \mathrm{~g} \leq e \leq 2 \mathrm{~g} \\
5 \mathrm{~g} \leq e\end{array}$ & $\begin{array}{l}100 \\
500\end{array}$ & $\begin{array}{l}10000 \\
10000\end{array}$ & $\begin{array}{l}20 e \\
20 e\end{array}$ \\
\hline Ordinaria (IIII) & $5 \mathrm{~g} \leq e$ & 50 & 1000 & $10 e$ \\
\hline
\end{tabular}

TABLA 2

ERRORES MÁXIMOS PERMITIDOS (emp) PARA LA VERIFICACIÓN SUBSECUENTE [2]

\begin{tabular}{ccccc}
\hline $\begin{array}{c}\text { Errores máximos } \\
\text { permitidos para } \\
\text { la verificación } \\
\text { subsecuente }\end{array}$ & Clase (I) & Clase (II) & Clase (III) & Clase (IIII) \\
\hline $\pm 1 e$ & $0<m<50000$ & $0<m<5000$ & $0<m<500$ & $0<m<50$ \\
$\pm 2 e$ & $50000<m<200000$ & $5000<m<20000$ & $500<m<2000$ & $50<m<200$ \\
$\pm 3 e$ & $200000<m$ & $20000<m<100000$ & $2000<m<10000$ & $200<m<1000$ \\
\hline
\end{tabular}

Nota: Para mayor información acerca de la clasificación de los IPFNA y sus errores máximos permitidos, revisar la OIML R76 [2].

Por ejemplo, un instrumento clase (I) de las siguientes características,

Max $=1 \mathrm{~kg}$

$\operatorname{Min}=0.1 \mathrm{~g}$

$d=e=1 \mathrm{mg}$

$n=1000000$

Es decir, tendría los siguientes errores máximos permitidos:

$\pm 1 \mathrm{mg}$, en el intervalo de $100 \mathrm{mg}$ a $50 \mathrm{~g}$

$\pm 2 \mathrm{mg}$, en el intervalo de $50.001 \mathrm{~g}$ a $200 \mathrm{~g}$

$\pm 3 \mathrm{mg}$, en el intervalo de $200.001 \mathrm{~g} \mathrm{a} 1 \mathrm{~kg}$ 
Por tanto, una indicación del instrumento anterior igual a $130 \mathrm{~g}$ se espera que no tenga un error mayor a $\pm 2 \mathrm{mg}$. Sin embargo, el intervalo formado por la indicación y el error máximo permitido (positivo y negativo) del instrumento que queda de la siguiente manera: [129.998 g, $130.002 \mathrm{~g}$ ], ¿cubre el valor verdadero del mensurando? La respuesta es que podría incluirlo, pero con un nivel de confianza de aproximadamente el $45 \%$.

\section{CALIBRACIÓN DE LOS IPFNA}

El uso de instrumentos para pesar (balanzas), usualmente empleados en actividades relacionadas con el control de calidad de productos y procesos, no necesariamente sujetas a control metrológico legal, y que por tanto, no requieren verificación legal, motivó el desarrollo de Guías Técnicas de Calibración de este tipo de instrumentos [6,7] con la intención de que el usuario conozca el error e incertidumbre asociada a la indicación del instrumento cuando lo utiliza, una vez que ha sido calibrado.

La calibración de un IPFNA consiste básicamente en:

- la aplicación de cargas de prueba al instrumento para pesar bajo condiciones especificadas,

- la determinación del error o sesgo de la indicación, y

- la estimación de la incertidumbre de la medición a ser atribuida a los resultados.

Las pruebas principales que se le realizan a un IPFNA en calibración son excentricidad, repetibilidad y la prueba de error de indicación. Las mismas se realizan con patrones de masa calibrados y certificados; en general, el metrólogo debe confirmar la compatibilidad de las necesidades del usuario del instrumento (incertidumbre requerida) con las características de las pesas (clase de exactitud, incertidumbre, entre otras).

El modelo de medición que se utiliza para la calibración es el siguiente [6,7]:

$$
E=I-m_{\text {ref }}
$$

$E$ es el error de indicación del instrumento bajo prueba, $I$ es la indicación del instrumento y $m_{\text {ref }}$ la masa de referencia.

Por su parte, la indicación del instrumento $I$ tiene el siguiente modelo,

$$
I=I_{\mathrm{L}}+\delta I_{\text {digL }}+\delta I_{\text {rep }}+\delta I_{\text {ecc }}-I_{0}-\delta I_{\text {digo }}
$$

En donde,

$I_{\mathrm{L}} \quad$ es la indicación del instrumento con carga (pesa patrón).

$I_{0} \quad$ es la indicación del instrumento sin carga 
$\delta I_{\text {digt }}$ es la corrección debida a la resolución finita del instrumento, cuando tiene la carga sobre el receptor.

$\delta I_{\text {dig0 }}$ es la corrección debida a la resolución finita del instrumento cuando no tiene carga.

$\delta I_{\text {rep }}$ es la corrección debida a la repetibilidad del instrumento.

$\delta I_{\text {ecc }}$ es la corrección debida a la excentricidad del instrumento.

Por otro lado, la masa de referencia, se estima de la siguiente manera,

$$
m_{\mathrm{ref}}=m_{\mathrm{N}}+\delta m_{\mathrm{c}}+\delta m_{\mathrm{B}}+\delta m_{\mathrm{D}}+\delta m_{\mathrm{conv}}+\delta m_{\ldots}
$$

En donde,

$m_{\mathrm{N}} \quad$ es el valor nominal de las pesas patrón.

$\delta m_{\mathrm{C}} \quad$ es la corrección en masa convencional de las pesas patrón.

$\delta m_{\mathrm{B}} \quad$ es la corrección debida al empuje del aire.

$\delta m_{\mathrm{D}} \quad$ es la corrección debida a la posible deriva de las pesas patrón.

$\delta m_{\text {conv }}$ es la corrección debido a los efectos de convección (diferencias de temperatura).

$\delta m \ldots$ son correcciones que pueden deberse a efectos adicionales de la calibración en particular.

Por otro lado, en algunas calibraciones no es posible llevarla a cabo solo con pesas, y es necesario emplear cargas de sustitución; esto debe ser considerado en la evaluación de la incertidumbre, pues viene ampliamente desarrollada en [6,7].

\section{USO DEL IPFNA CALIBRADO}

Una vez que el instrumento ha sido calibrado, se conocen los errores de indicación (y por tanto la corrección), y sus incertidumbres asociadas, información que es comunicada al usuario en el certificado de calibración correspondiente por el proveedor del servicio de calibración. Al instrumento en su uso normal, al tener una nueva indicación, se agregan nuevas contribuciones al modelo de medición. El modelo de medición de un valor de masa (masa convencional) de un resultado de pesada $(W)$ utilizando un instrumento calibrado es el siguiente $[6,7]$ :

$$
W=R+\delta R_{\mathrm{digL}}+\delta R_{\mathrm{rep}}-R_{0}-\delta R_{\mathrm{dig} 0}-E
$$

Aquí, 
E es el error asociado a la indicación en uso del instrumento.

$R \quad$ es la indicación con carga del instrumento (calibrado) en uso normal.

$R_{0} \quad$ es la indicación sin carga del instrumento (calibrado) en uso normal.

$\delta R_{\text {digL }}$ es la corrección debida a la resolución finita del instrumento, cuando tiene la carga sobre el receptor.

$\delta R_{\text {digo }}$ es la corrección debida a la resolución finita del instrumento cuando no tiene carga.

$\delta R_{\text {rep }}$ es la corrección debida a la repetibilidad del instrumento.

Es posible aplicar el uso normal del instrumento calibrado sin aplicar corrección alguna a la indicación (obtener un valor de pesada, $W$ ), por lo que la incertidumbre asociada con el valor de pesada debe contener toda la información necesaria para incluir el valor verdadero del mensurando. Es decir, una incertidumbre global expandida $U_{\mathrm{gl}}(W)$,

$$
W=R \pm U_{\mathrm{gl}}(W)
$$

El valor sería trazable al Sistema Internacional de Unidades siempre que la incertidumbre global incluya todas las fuentes de incertidumbre correspondientes a la calibración y al uso posterior del instrumento.

\section{ESTIMACIÓN DE LA INCERTIDUMBRE DE UN IPFNA VERIFICADO}

Si el IPFNA tiene aprobación de modelo y ha sido verificado de acuerdo a la OIML R76, se puede estimar la incertidumbre asociada con una indicación cualquiera. Lo anterior con base en el modelo de medición del valor de pesada de un instrumento calibrado, el cual tiene asociada una incertidumbre global. La estimación de la incertidumbre del valor de pesada de un instrumento verificado se estima de acuerdo con las características propias del instrumento, así como a los emp del mismo acorde a la clase de exactitud de este.

Respecto al cálculo de la estimación de la incertidumbre global de la indicación de un instrumento verificado, se aplica la ley de propagación de incertidumbres a las ecuaciones 1, 2, 3 y 4 [9]. La incertidumbre estándar global del valor de pesada de un IPFNA verificado puede aproximarse a la siguiente expresión, para ello es importante igualar las incertidumbres asociadas a las indicaciones de la balanza en calibración y en uso normal, $u\left(\delta I_{x}\right)=u\left(\delta R_{x}\right)$,

$$
\begin{aligned}
& u_{\mathrm{gl}}(W)=\left\{2\left[u^{2}\left(\delta I_{\mathrm{digL}}\right)+u^{2}\left(\delta I_{\mathrm{dig} 0}\right)+u^{2}\left(\delta I_{\text {rep }}\right)\right]+u^{2}\left(\delta I_{\mathrm{ecc}}\right)+u^{2}(E)++u^{2}\left(\delta m_{\mathrm{c}}\right)+u^{2}\left(\delta m_{\mathrm{B}}\right)+\right. \\
& \left.u^{2}\left(\delta m_{D}\right)+u^{2}\left(\delta m_{\text {conv }}\right)\right\}^{\frac{1}{2}}
\end{aligned}
$$


En donde,

$u_{\mathrm{gl}}(W) \quad$ es la incertidumbre estándar global asociada al valor de pesada del instrumento verificado.

$u\left(\delta I_{\text {digL }}\right)$ es la incertidumbre estándar debida la resolución del instrumento cuando tiene la carga sobre el receptor de carga.

$u\left(\delta I_{\text {digo }}\right)$ es la incertidumbre estándar debida la resolución del instrumento sin carga.

$u\left(\delta I_{\text {rep }}\right)$ es la incertidumbre estándar asociada a la falta de repetibilidad de las indicaciones del instrumento.

$u\left(\delta I_{\text {ecc }}\right) \quad$ es la incertidumbre estándar debida al efecto de la excentricidad en la indicación del instrumento.

$u(E) \quad$ es la incertidumbre asociada al error de indicación del instrumento.

$u\left(\delta m_{\mathrm{C}}\right)$ es la incertidumbre estándar asociada a la variabilidad de la masa convencional de los patrones utilizados en la verificación del instrumento.

$u\left(\delta m_{\mathrm{B}}\right)$ es la incertidumbre debido al efecto del empuje del aire sobre los patrones de masa en la verificación.

$u\left(\delta m_{\mathrm{D}}\right)$ es la incertidumbre asociada a la deriva de la masa (convencional) de los patrones utilizados en la verificación desde su última calibración.

$u\left(\delta m_{\text {conv }}\right)$ es la incertidumbre asociada a los efectos de convección debido a la diferencia de temperatura entre las pesas y el instrumento al realizar la verificación de este.

La incertidumbre global expandida $U_{\mathrm{gl}}(W)$ a un nivel de confianza de, aproximadamente, el $95 \%$ se obtiene al multiplicar la incertidumbre estándar combinada (global) por un factor de cobertura $k=2$.

$$
U_{\mathrm{gl}}(W)=k u_{\mathrm{gl}}(W)
$$

Los valores de las incertidumbres pueden ser estimados considerando las características de fabricación del instrumento (p.ej. resolución, alcance), y los emp del instrumento en relación con su indicación en todo el alcance, así como en condiciones de excentricidad y repetibilidad, y que corresponden a la clase de exactitud propia del instrumento. Así, la incertidumbre asociada con el error de indicación del instrumento $u(E)$ se puede estimar al asumir el emp del instrumento como un medio intervalo de una distribución de probabilidad rectangular,

$$
u(E) \cong \frac{e m p}{\sqrt{3}}
$$


La incertidumbre estándar debida a la resolución finita del instrumento cuando tiene la carga sobre el receptor de carga $u\left(\delta I_{\mathrm{digL}}\right)$ se estima con la siguiente expresión. Aquí, $d$ es la resolución del instrumento y a la cual se le asigna una distribución de probabilidad rectangular,

$$
u\left(\delta I_{\text {digL }}\right)=\frac{d}{2 \sqrt{3}}
$$

Respecto a la incertidumbre de la resolución del instrumento sin carga $u\left(\delta I_{\text {digL }}\right)$, esta puede ser aproximada a la siguiente expresión, considerando que el instrumento debe tener aprobación de modelo de acuerdo a la OIML R76, en donde $d_{0}$ es la resolución del instrumento sin carga, y la cual tiene asociada un intervalo de probabilidad rectangular de la mitad de la resolución en el resto del alcance del instrumento,

$$
u\left(\delta I_{\operatorname{dig} 0}\right) \cong \frac{d_{0}}{4 \sqrt{3}}
$$

La incertidumbre relacionada con la repetibilidad del instrumento se estima como la desviación estándar de las indicaciones del instrumento $s(I)$, la cual se aproxima al emp correspondiente del instrumento,

$$
u\left(\delta I_{\text {rep }}\right)=s(I) \cong e m p
$$

En cuanto a la incertidumbre de la excentricidad del instrumento, esta puede estimarse de la siguiente manera,

$$
u\left(\delta I_{\mathrm{ecc}}\right) \cong \frac{e m p}{\sqrt{3}}
$$

Por su parte, para considerar la incertidumbre asociada con la variabilidad de la masa convencional de las pesas patrón que se utilizaron para verificar el instrumento se toma en cuenta que el emp de la pesa no debe ser mayor a 1/3 del emp del instrumento verificado,

$$
u\left(\delta m_{\mathrm{c}}\right) \cong \frac{e m p}{3 \sqrt{3}}
$$

Además, la incertidumbre debido al empuje del aire puede expresarse en términos del emp del instrumento de la siguiente manera:

$$
u\left(\delta m_{\mathrm{B}}\right) \cong \frac{e m p}{12 \sqrt{3}}
$$

Por otro lado, la incertidumbre debida a la deriva de los patrones de las pesas expresado en términos del emp del instrumento quedaría así:

$$
u\left(\delta m_{\mathrm{D}}\right) \cong \frac{e m p}{18 \sqrt{3}}
$$


Finalmente, la incertidumbre por los efectos de convección (diferencia de temperatura entre las pesas patrón y el instrumento) se desprecia, asumiendo que las pesa se dejaron ambientar previo a la ejecución de las pruebas de verificación:

$$
u\left(\delta m_{\text {conv }}\right) \approx 0
$$

Sustituyendo las incertidumbres anteriores en la expresión (6), simplificando y multiplicando por un factor de cobertura $k=2$, se tiene la incertidumbre global expandida $U_{\mathrm{gl}}(W)$, asociada al valor de pesada del instrumento verificado,

$$
U_{\mathrm{gl}}(W) \approx 2 \sqrt{0.208 e^{2}+2.707 e m p^{2}}
$$

Tomando en cuenta que los instrumentos usualmente tienen tres escalones en lo que se refiere a los emp (ver tabla 2), los cuales corresponden a $1 e, 2$ e y $3 e$. La incertidumbre global de un valor de pesada expresada en términos de la división de la escala de verificación del instrumento se presenta en la Tabla 3.

TABLA 3

INCERTIDUMBRE EXPANDIDA GLOBAL ASOCIADA CON EL VALOR DE PESADA DE UN INSTRUMENTO VERIFICADO, EXPRESADA COMO UN MÚLTIPLO DE LA DIVISIÓN DE LA ESCALA DE VERIFICACIÓN

\begin{tabular}{cc}
\hline $\boldsymbol{e m p} \boldsymbol{p}$ & $\boldsymbol{U}_{\boldsymbol{g l}}(\boldsymbol{W}), \boldsymbol{k}=\mathbf{2}$ \\
\hline Primer escalón & $3.4 e$ \\
$1 e$ & \\
Segundo escalón & $6.6 e$ \\
$2 e$ & \\
Tercer escalón & $9.9 e$ \\
$3 e$ & \\
\hline
\end{tabular}

Consecuentemente, la incertidumbre ligada a las indicaciones de los instrumentos para las cuatro clases de exactitud queda de la siguiente manera. Las líneas rojas representan el emp del instrumento en función de la carga expresada en términos de la división de la escala de verificación,e.

La recomendación internacional OIML R76 permite reducir el uso de carga de sustitución en la verificación de instrumentos para pesar de alto alcance de medición bajo ciertas condiciones. Por ejemplo, se puede utilizar el $50 \%$ del Max del instrumento en pesas patrón, o el $30 \%$ del Max si el error de repetibilidad del instrumento es igual o menor a $0.3 e$, en incluso puede reducirse la cantidad de patrones hasta el 20\% del Max si el error de repetibilidad del instrumento es igual o menor a $0.2 e$.

Para estimar la incertidumbre de la indicación del instrumento verificado con el uso de cargas de sustitución se pueden hacer varias aproximaciones razonables; sin embargo, la consideración 

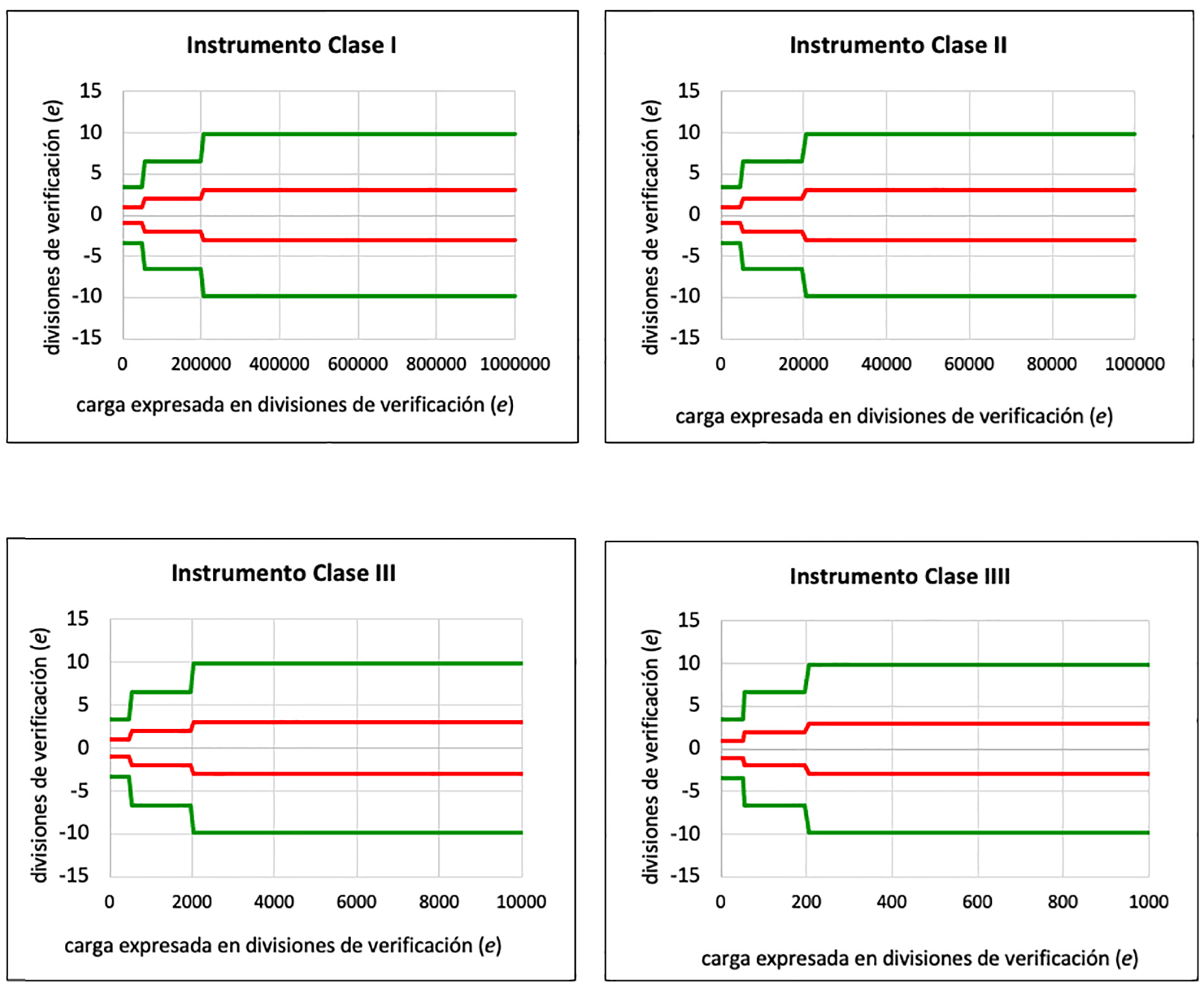

Figura 1. Incertidumbre expandida (en términos de $e$ ) asociada con la indicación de un instrumento para pesar de funcionamiento no automático en función de la carga expresada en términos de $e$ para las cuatro clases de exactitud de los instrumentos.

Nota. Las líneas verdes representan la incertidumbre expandida con $k=2$ y las líneas rojas el error máximo permitido, para las cuatro clases de exactitud.

que se hace en este trabajo es considerar que la repetibilidad puede tener un valor de $1 /{ }_{n}$ del emp correspondiente a la repetibilidad (y a la carga) para cuando se usan cargas de sustitución (siendo $1 /{ }_{n}$ el porcentaje de pesas patrón utilizado, p.ej. $0.5 \mathrm{emp}$ (rep) para el $50 \%$ de pesas patrón, 0.33 emp (rep) para el $33 \%$ de pesas patrón, etc.). Adicionalmente, se deben hacer algunas consideraciones adicionales, la varianza ${ }^{1}$ de la masa convencional de las pesas patrón se sustituye por la siguiente expresión,

$$
u^{2}\left(\delta m_{\mathrm{c}}\right) \approx u^{2}\left(L_{\mathrm{T}_{n}}\right)=N^{2} u^{2}\left(m_{\mathrm{c} 1}\right)+2\left[(N-1) u^{2}(I)\right]
$$

1. La varianza se obtiene al elevar al cuadrado la incertidumbre. 
En donde,

$N \quad$ es la cantidad de veces que se coloca la totalidad de los patrones de masa $(N>1$, se utiliza $N$ mayúscula para no confundirla con $n$ minúscula que en este trabajo se utiliza para el número de divisiones de verificación).

$u\left(m_{\mathrm{c1}}\right) \quad$ es la incertidumbre de la masa convencional de los patrones de masa.

$u(I) \quad$ es la incertidumbre combinada de la indicación.

La incertidumbre (estándar) combinada de la indicación del instrumento se estima con la siguiente ecuación,

$$
u(I)=\left[u^{2}\left(\delta I_{\text {digL }}\right)+u^{2}\left(\delta I_{\text {dig } 0}\right)+u^{2}\left(\delta I_{\text {rep }}\right)+u^{2}\left(\delta I_{\text {ecc }}\right)\right]^{\frac{1}{2}}
$$

Por otro lado, la incertidumbre de la masa de referencia cambia, pues la cantidad de patrones estaría limitada a un porcentaje del Max del instrumento. Por tanto, las incertidumbres relacionadas con las pesas patrón se pueden estimar fijas en función de la división del instrumento en lugar de variables y en función del error máximo permitido.

Además, la incertidumbre de la masa convencional del patrón, la incertidumbre del empuje del aire y la incertidumbre de la deriva del patrón se estimarían de la siguiente manera respectivamente,

$$
\begin{aligned}
& u\left(m_{\mathrm{c} 1}\right) \cong \frac{2 d}{3 \sqrt{3}} \\
& u\left(\delta m_{\mathrm{B}}\right) \cong \frac{d}{6 \sqrt{3}} \\
& u\left(\delta m_{\mathrm{D}}\right) \cong \frac{d}{9 \sqrt{3}}
\end{aligned}
$$

Por tanto, si se sustituyen las ecuaciones anteriores en (6), se puede estimar la incertidumbre global asociada con la indicación de un instrumento verificado acorde a la OIML R76 para el cual se utilizaron cargas de sustitución. Esta incertidumbre global expresada como una incertidumbre expandida puede aproximarse a la siguiente expresión:

$$
U_{\mathrm{gl}}(W) \cong 2 \sqrt{\left[0.148 N^{2}+0.208 N+0.013\right] e^{2}+[0.667 N+2] e m p^{2}}
$$


TABLA 4

INCERTIDUMBRE EXPANDIDA GLOBAL ASOCIADA CON EL VALOR DE PESADA DE UN INSTRUMENTO VERIFICADO CON EL USO DE CARGAS DE SUSTITUCIÓN, EXPRESADA COMO UN MÚLTIPLO DE LA DIVISIÓN DE LA ESCALA DE VERIFICACIÓN $e$.

\begin{tabular}{|c|c|c|c|c|}
\hline \% Pesas patrón & $\begin{array}{c}\text { Repetibilidad } \\
\text { emp }\end{array}$ & $\begin{array}{c}\text { Primer escalón } \\
1 e \\
U_{\mathrm{gl}}(\mathrm{W}), k=\mathbf{2}\end{array}$ & $\begin{array}{c}\text { Segundo escalón } \\
2 e \\
U_{\mathrm{gl}}(\mathrm{W}), k=2\end{array}$ & $\begin{array}{c}\text { Tercer escalón } \\
3 e \\
U_{\mathrm{gl}}(\mathrm{W}), k=2\end{array}$ \\
\hline $100 \%$ & 1.00 & $3.4 \mathrm{e}$ & $6.6 e$ & $9.9 e$ \\
\hline $50 \%$ & 0.50 & $3.5 e$ & $6.6 e$ & $11.1 e$ \\
\hline $30 \%$ & 0.30 & $3.5 e$ & $7.6 e$ & $12.3 e$ \\
\hline $25 \%$ & 0.25 & $3.5 e$ & $7.6 e$ & $13.5 e$ \\
\hline $20 \%$ & 0.20 & $3.5 e$ & $8.5 e$ & $14.5 e$ \\
\hline
\end{tabular}

Como ejemplo se presenta una gráfica de la incertidumbre relacionada con la indicación de un instrumento para pesar clase III, el cual fue verificado con el uso de cargas de sustitución.

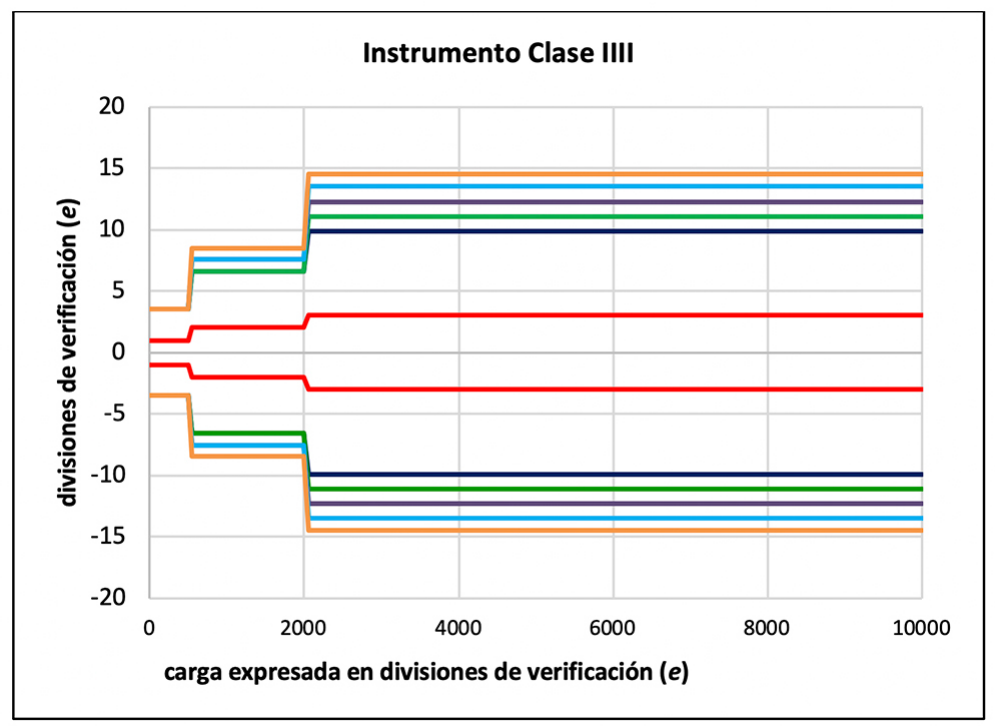

Fig. 2. Incertidumbre expandida relacionada con la indicación un instrumento para pesar clase III verificado con el uso de cargas de sustitución.

Nota. La incertidumbre está expresada en términos de la división de la escala de verificación $e$, y en función de la carga también expresada en términos de $e$. Las líneas rojas centrales representan el error máximo permitido y las líneas de colores la incertidumbre asociada la indicación del instrumento; las más cercanas al emp (líneas azul oscuro) se refieren al instrumento verificado solo con patrones, $100 \%$ de pesas patrón, y las más alejadas se refieren a aquellas en las cuales se utilizaron más enlaces o menor cantidad de pesas patrón, $50 \%, 30 \%, 25 \%$ y $20 \%$ respectivamente. Todas las incertidumbres son expandidas $\operatorname{con} k=2$. 


\section{EJEMPLO}

Un instrumento para pesar de funcionamiento no automático Clase I con las siguientes características:

Max $=1 \mathrm{~kg}, d=1 \mathrm{mg}$ (igual a $e$ ), $n=1000$ 000. Este ha sido verificado de acuerdo a la OIML R76. Además, presenta algunos errores máximos permitidos e incertidumbre expandida de la indicación, en función de la carga (ver Tabla 5).

TABLA 5

\begin{tabular}{ccc}
$\begin{array}{c}\text { ERRORES MÁXIMOS PERMITIDOS E INCERTIDUMBRE EXPANDIDA ASOCIADA } \\
\text { CON LA INDICACIÓN DEL INSTRUMENTO EN MEDICIÓN, EN FUNCIÓN DE LA CAR } \\
\text { SOBRE SU RECEPTOR }\end{array}$ \\
$\begin{array}{ccc}\text { Carga del } \\
\text { instrumento, } \boldsymbol{m}\end{array}$ & $\begin{array}{c}\text { Error máximo } \\
\text { permitido, } \boldsymbol{e m p}\end{array}$ & $\begin{array}{c}\text { Inc. global expandida asociada } \\
\text { con la indicación del } \\
\text { instrumento, } \boldsymbol{U}_{\mathrm{gl}}(\boldsymbol{W}), \boldsymbol{k}=\mathbf{2}\end{array}$ \\
\hline $0 \mathrm{~g}<m<50 \mathrm{~g}$ & $\pm 1 \mathrm{mg}$ & $3.4 \mathrm{mg}$ \\
$50 \mathrm{~g}<m<200 \mathrm{~g}$ & $\pm 2 \mathrm{mg}$ & $6.6 \mathrm{mg}$ \\
$200 \mathrm{~g}<m<1000 \mathrm{~g}$ & $\pm 3 \mathrm{mg}$ & $9.9 \mathrm{mg}$ \\
\hline
\end{tabular}

Por tanto, una indicación del instrumento de $205 \mathrm{~g}$ implica que el valor de la masa convencional del objeto pesado tendría un valor de incertidumbre asociado de $U_{\mathrm{gl}}(W)=9.9 \mathrm{mg}(k=2)$, lo que implica que el valor de masa convencional estaría comprendido en el siguiente intervalo con una probabilidad de aproximadamente el $95 \%$, [204.990 1 g, $205.0099 \mathrm{~g}$ ].

Por otro lado, se tiene un instrumento Clase III con las siguientes características: Max $=80000$ $\mathrm{kg},=20 \mathrm{~kg}$ (igual a $e$ ), $n=4000$. Esto se verificó con el $30 \%$ de pesas patrón. A su vez, tendría los siguientes errores máximos permitidos e incertidumbre expandida asociada a la indicación, en función de la carga (ver Tabla 6).

TABLA 6

ERRORES MÁXIMOS PERMITIDOS E INCERTIDUMBRE EXPANDIDA ASOCIADA CON LA INDICACIÓN DEL INSTRUMENTO DE MEDICIÓN, EN FUNCIÓN DE LA CARGA SOBRE SU RECEPTOR

\begin{tabular}{ccc}
\hline Carga del instrumento, $\boldsymbol{m}$ & $\begin{array}{c}\text { Error máximo } \\
\text { permitido, } \boldsymbol{e m p} \boldsymbol{p}\end{array}$ & $\begin{array}{c}\text { Inc. global expandida asociada } \\
\text { la indicación del } \\
\text { instrumento, } \boldsymbol{U}_{\mathrm{gl}}(\boldsymbol{W}), \boldsymbol{k}=\mathbf{2}\end{array}$ \\
\hline $0 \mathrm{~kg}<m<10000 \mathrm{~kg}$ & $\pm 20 \mathrm{~kg}$ & $70 \mathrm{~kg}$ \\
$10000 \mathrm{~kg}<m<40000 \mathrm{~kg}$ & $\pm 40 \mathrm{~kg}$ & $152 \mathrm{~kg}$ \\
$40000 \mathrm{~kg}<m<80000 \mathrm{~kg}$ & $\pm 60 \mathrm{~kg}$ & $247 \mathrm{~kg}$ \\
\hline
\end{tabular}


Por tanto, una indicación del instrumento de $45300 \mathrm{~kg}$ tendría asociado un valor de incertidumbre de $U_{\mathrm{gl}}(W)=247 \mathrm{~kg}(k=2)$. Lo anterior implica que el valor de la masa convencional de la carga sobre el instrumento estaría dentro del siguiente intervalo [45 $053 \mathrm{~kg}, 45547 \mathrm{~kg}$ ], con un $95 \%$ de nivel de confianza.

\section{CONCLUSIONES}

La incertidumbre de la indicación de un instrumento verificado de acuerdo a la OIML R76 puede ser estimada con la ecuación (17), la cual está en función de la resolución del instrumento y del error máximo permitido del instrumento correspondiente a la carga, la cual es de aproximadamente 3.4 veces el emp para el primer escalón del instrumento y de 3.3 veces el emp correspondiente al segundo y tercer escalón (ver Tabla 3 y figura 1).

En instrumentos para pesar de alto alcance de medición, los cuales han sido verificados utilizando cargas de sustitución, la incertidumbre relacionada con la indicación del instrumento es de, aproximadamente, 3.5 veces el emp en el primer escalón, hasta 4.2 veces el emp en el segundo escalón y hasta 4.8 veces el emp en el tercer escalón del instrumento (ver Tabla 4).

Por tanto, si se calcula la probabilidad de que el valor de masa (convencional) de un objeto o carga pesado/a en un instrumento para pesar verificado, la probabilidad de que el valor "verdadero" de la masa convencional se encuentre dentro del intervalo del emp, [emp $\left.{ }^{+}, e m p^{-}\right]$va del $44.2 \%$ para el primer escalón ( $1 e$ ) hasta el $45.5 \%$ de nivel de confianza para el tercer escalón $(3 e)$, cuando el instrumento es verificado sólo con pesas patrón. Por otro lado, si se utilizan cargas de sustitución, esta probabilidad puede ser desde $43.4 \%$ para el primer escalón hasta el $32 \%$ en el tercer escalón (cuando se utilizan sólo el $20 \%$ de pesas patrón en la verificación) (ver tabla 7).

TABLA 7

RESUMEN DE LA INCERTIDUMBRE GLOBAL ASOCIADA CON LA INDICACIÓN DE UN INSTRUMENTO VERIFICADO EXPRESADA EN MÚLTIPLOS DEL $е m p$, EL VALOR DE LA $t$ DE STUDENT Y LA PROBABILIDAD ASOCIADA CORRESPONDIENTE AL INTERVALO DEL $e m p$

DE UNA DISTRIBUCIÓN DE PROBABILIDAD CON MEDIA 0 Y DESVIACIÓN ESTÁNDAR IGUAL A LA INCERTIDUMBRE ESTÁNDAR GLOBAL, $u_{\mathrm{gl}}(k=1)$

\begin{tabular}{|c|c|c|c|c|c|c|c|c|c|}
\hline \multirow{2}{*}{$\begin{array}{c}\% \text { pesas } \\
\text { patrón en } \\
\text { verificación }\end{array}$} & \multicolumn{3}{|c|}{ 1er escalón $( \pm 1 e)$} & \multicolumn{3}{|c|}{ 2do escalón $( \pm 2 e)$} & \multicolumn{3}{|c|}{ 3er escalón $( \pm 3 e)$} \\
\hline & $\begin{array}{c}U_{\mathrm{gl}}(W) \\
(\text { emp })\end{array}$ & $\begin{array}{c}t \\
\text { de student }\end{array}$ & $1-\alpha$ & $\begin{array}{c}U_{\mathrm{gl}}(W) \\
(\text { emp }\end{array}$ & $\begin{array}{c}t \\
\text { de student }\end{array}$ & $1-\alpha$ & $\begin{array}{c}U_{\mathrm{gl}}(W) \\
(e m p)\end{array}$ & $\begin{array}{c}t \\
\text { de student }\end{array}$ & $1-\alpha$ \\
\hline $100 \%$ & 3.4 & 0.59 & $44.2 \%$ & 3.3 & 0.60 & $45.3 \%$ & 3.3 & 0.61 & $45.5 \%$ \\
\hline $50 \%$ & 3.5 & 0.57 & $43.4 \%$ & 3.3 & 0.60 & $45.3 \%$ & 3.7 & 0.54 & $41.0 \%$ \\
\hline $30 \%$ & 3.5 & 0.57 & $43.4 \%$ & 3.8 & 0.53 & $40.2 \%$ & 4.1 & 0.49 & $37.3 \%$ \\
\hline $25 \%$ & 3.5 & 0.57 & $43.4 \%$ & 3.8 & 0.53 & $40.2 \%$ & 4.5 & 0.45 & $34.4 \%$ \\
\hline $20 \%$ & 3.5 & 0.57 & $43.4 \%$ & 4.2 & 0.47 & $36.3 \%$ & 4.8 & 0.41 & $32.0 \%$ \\
\hline
\end{tabular}


De los datos de la Tabla 7, en el mejor de los casos, la probabilidad de encontrar el valor de masa dentro de un intervalo centrado en la indicación de un instrumento verificado y con límites comprendidos entre \pm emp del instrumento es menor al $50 \%$.

Conocer la incertidumbre de medición global asociada con la indicación de instrumentos para pesar verificados puede ser de mucha utilidad para la toma de decisiones que involucran el uso de dichos instrumentos.

\section{REFERENCIAS}

[1] International Organization for Standardization (2003) ISO 10012:2003 Sistemas de gestión de las mediciones - Requisitos para los procesos de medición y los equipos de medición.

[2] Organisation Internationale de Métrologie Légale, (2006) OIML R 76-1 Edition 2006. Non-automatic weighing instruments - Part 1: Metrological and technical requirements - Tests. Organisation Internationale de Métrologie Légale. https://www.oiml.org/en/files/pdf_r/r076-p-e06.pdf

[3] Norma Oficial Mexicana (1994) NOM-010-SCFI-1994 "Instrumentos de medición - Instrumentos para pesar de funcionamiento no automático - Requisitos técnicos y metrológicos”. Diario Oficial de la Federación, 9 de junio de 1999.

[4] Proyecto de Norma Oficial Mexicana (2018) PROY-NOM-010-SCFI-2017, Instrumentos de medición-Instrumentos para pesar de funcionamiento no automático-Requisitos técnicos y metrológicos, métodos de prueba y de verificación (cancela al PROY-NOM-010-SCFI-2014 y cancelará a la NOM-010-SCFI-1994)., Diario Oficial de la Federación, 1 de agosto de 2018. https://dof.gob.mx/ nota_detalle.php?codigo $=5533602 \&$ fecha $=01 / 08 / 2018$

[5] Ley de Infraestructura de la Calidad, Diario Oficial de la Federación el 1 de julio de 2020. http://www. diputados.gob.mx/LeyesBiblio/pdf/LICal_010720.pdf

[6] Sistema Interamericano de Metrología (2009), SIM Guidelines on the calibration of non-automatic weighing instruments - SIM MWG/cg-01/v.00, 2009. https://www.cenam.mx/publicaciones/gratuitas/descarga/default.aspx?arch=/pdf_calibracion/SIM_MWG7_cg-01_v00.pdf

[7] THE EUROPEAN ASSOCIATION OF NATIONAL METROLOGY INSTITUTES (2015), EURAMET Guidelines on the Calibration of Non-Automatic Weighing Instruments I TC-M I Version 4.0, 11/2015 https://www.euramet.org/publications-media-centre/calibration-guidelines/

[8] Organisation Internationale de Métrologie Légale, (2004) OIML R 111-1 Edition 2004, Weights of classes $\mathrm{E}_{1}, \mathrm{E}_{2}, \mathrm{~F}_{1}, \mathrm{~F}_{2}, \mathrm{M}_{1}, \mathrm{M}_{1-2}, \mathrm{M}_{2}, \mathrm{M}_{2-3}$ and $\mathrm{M}_{3}$ - Part 1: Metrological and technical requirements. https://www.oiml.org/en/files/pdf_r/r111-p-e04.pdf

[9] Joint Committee for Guides in Metrology (2008) JCGM 100:2008, Evaluation of measurement data - Guide to the expression of uncertainty in measurement - First edition September 2008. https:// www.bipm.org/utils/common/documents/jcgm/JCGM_100_2008_E.pdf

[10] Joint Committee for Guides in Metrology (2012) JCGM 106:2012, Evaluation of measurement data The role of measurement uncertainty in conformity assessment - First edition October 2012. https:// www.bipm.org/utils/common/documents/jcgm/JCGM_106_2012_E.pdf 